\title{
Die kausalen Verknüpfungsmittel des Deutschen und des Italienischen. Eine kontrastive Beschreibung unter formalem und funktionalem Aspekt*
}

\author{
Sabrina Ballestracci (Florenz)
}

\begin{abstract}
This paper is a contrastive description of the causal grammatical structures mentioned in five grammar books of German and four grammar books of Italian. The study is inspired by the results of a previous empirical research upon the causal connectors denn, weil, da in German and perché, poiché, siccome in Italian, which has demonstrated that there are many similarities, but without any complete correspondence between the analyzed German and Italian grammatical structures. The purpose of this work is to verify if this thesis holds true not only for a limited number of connectors, but also for the whole system of German and Italian causal structures. The study is based upon a broad concept of causality, seen as the question about "why and with which purpose do events und states happen and verify". It focuses not only on the grammatical structures which are traditionally defined causal, but also on final and conditional structures. The results of the research are summarized at the end of the paper with the expectation that they will be helpful to the didactic transmission of the German causal structures with Italian learners.
\end{abstract}

\section{$1 \quad$ Zur Einführung}

Die bisher durchgeführten empirischen Untersuchungen zum Erwerb des Deutschen als Fremdsprache postulieren die Gültigkeit und Nützlichkeit der kontrastiven Methode im DaFUnterricht für die Entwicklung der grammatischen bzw. metasprachlichen Kenntnisse der DaF-Lerner (Diehl et al. 2000; Brdar-Szabó 2001; Terrasi-Haufe 2004; Ballestracci 2009). Von dieser Annahme ausgehend, möchte ich die kausalen Verknüpfungsmittel des Deutschen und des Italienischen kontrastiv untersuchen, dies mit dem Ziel, Ähnlichkeiten und Unterschiede zwischen den beiden Sprachsystemen zu beschreiben. Dabei stütze ich mich auf die Ergebnisse einer Untersuchung zu den prototypischen kausalen Konnektoren denn, weil, da des Deutschen und perché, poiché, siccome des Italienischen, welche zeigen, dass "es zwischen den deutschen und den italienischen untersuchten Konnektoren vielfältige Ähnlichkeiten gibt, jedoch keine direkten Entsprechungen (Ravetto/Blühdorn 2011)".1

\footnotetext{
* An dieser Stelle danke ich Hardarik Blühdorn, Horst Sitta, den drei Moderatoren der 14. Arbeitsgruppe der Dritten Tagung Deutsche Sprachwissenschaft in Italien (Rom, Februar 2010) - Gabriela Perrig, Klaus Peter und Michael Schümann - sowie dem anonymen Gutachter für ihre zahlreichen und wertvollen Kommentare und Anregungen.

${ }^{1}$ Die sechs prototypischen Kausalkonjunktionen werden von Ravetto/Blühdorn (2011: 207-209) aufgrund eines Korpus, das 1'020 Beispielsätze aus allen Textgenres umfasst, vergleichend in syntaktischer und semantischer Hinsicht untersucht, und zwar unter folgenden Aspekten: 1) "der Gebrauch und das Verhalten der sechs Konjunktionen im Bezug auf die Informationsstruktur"; 2) "die mögliche Stellung des Teilsatzes, der die Ursache kodiert, in Bezug auf den Teilsatz, der die Wirkung ausdrückt"; 3) "die hierarchisch-syntaktischen Beziehungen zwischen den Teilsätzen (Unter- oder Nebenordnung)" (ebd.). Meine Analyse stützt sich nicht auf ein Korpus
} 
Über diese Ergebnisse hinaus werde ich aufgrund der Strukturen, die in fünf Grammatiken des Deutschen und vier Grammatiken des Italienischen beschrieben werden, das ganze Inventar der kausalen Verknüpfungsmittel der beiden Sprachen in den Blick nehmen und zeigen, dass die von Ravetto und Blühdorn aufgestellte These nicht nur für eine beschränkte Anzahl der kausalen Konnektoren des Deutschen und des Italienischen gilt, sondern für das ganze kausale System der beiden Sprachen: Das Italienische und das Deutsche besitzen ein approximativ äquivalentes Repertoire von grammatischen Strukturen zum Ausdruck der Kausalität, wobei sich grundsätzlich Entsprechungen zwischen den beiden Sprachen ergeben. Es gibt nur wenige Unterschiede, die vor allem auf der morphosyntaktischen Ebene auftreten, und zwar bei jenen Ausdrucksmitteln, die sich unter funktionalem Aspekt entsprechen und eine ähnliche, aber nichtidentische formale Struktur aufweisen.

Um das kausale System des Deutschen und des Italienischen zu beschreiben, ist ein Inventar der kausalen Verknüpfungsmittel der beiden Sprachen nötig. Dies gibt es noch nicht. Es wird im vorliegenden Beitrag auf der Basis der existierenden grammatischen Beschreibungen des Deutschen und des Italienischen zusammengestellt. Berücksichtigt werden dabei folgende Grammatiken: für das Deutsche Weinrich (1993), Zifonun et al. (1997), Eisenberg (2006), Engel (2009) und Duden (2009); für das Italienische Serianni (1991), Battaglia/Pernicone (1994), Dardano/Trifone (1995) und Renzi et al. (2001).

Auf der Basis der vorliegenden Untersuchung möchte ich nützliche Implikationen für die Grammatikvermittlung des Deutschen im DaF-Unterricht in Italien formulieren, dies in der Hoffnung, dass sie dazu beitragen können, didaktische Materialien zur Beschreibung der kausalen Strukturen des Deutschen zu entwickeln. Dabei gehe ich vom "integrierenden" didaktischen Ansatz aus, wonach im Unterricht die Analyse der formalen Struktur einer Sprache in direkte Verbindung mit der Analyse ihrer semantisch-funktionalen Valenz gestellt werden soll. Hierbei plädiere ich für eine grammatische Beschreibung, die ermöglicht, einerseits einen übersichtlichen Blick auf die Zielgrammatik zu erhalten (vgl. Rall 2001), andererseits auf die Besonderheiten der verschiedenen grammatischen Phänomene einzugehen. Um Übersichtlichkeit zu erreichen, wird Kausalität zuerst auf der semantischen Ebene definiert, und zwar als kausale Kohärenz, die eine breitere Definition von Kausalität bietet als jene, die üblicherweise in den traditionellen Grammatiken des Deutschen und des Italienischen vorgeschlagen wird. In der deutschen Grammatikschreibung wurden schon immer zwei unterschiedliche Begriffe von Kausalität verwendet, ein engerer und ein weiterer (vgl. dazu Blühdorn 2010). Gegenwärtig scheint weitherum der engere zu dominieren. Ich werde mit dem weiteren Begriff arbeiten, indem ich die Oberkategorie der "kausalen und semantisch verwandten Relationen" unterscheide, die ich Kausalität im weiteren Sinne nenne, zu der nicht nur die traditionell als "kausal" betrachteten Verhältnisse (hier als kausal im engeren Sinne bezeichnet), sondern auch die finalen und konditionalen Verhältnisse zählen. ${ }^{2}$ Bezogen darauf werde ich erläutern, inwieweit auch finale und konditionale Verhältnisse die Verknüpfung zweier Ereignisse bzw. Zustände bezeichnen, die in kausaler Relation stehen. Erst in einem zweiten Schritt werden kausale i. e. S., finale und konditionale Ausdrucksmöglichkeiten vereinzelt analysiert, und zwar mit dem Ziel, die formalen Besonderheiten jedes spezifischen Bereiches zu beschreiben.

von Beispielsätzen, sondern auf das Repertoire von Strukturen, die in fünf Grammatiken des Deutschen und vier Grammatiken des Italienischen beschrieben werden.

${ }^{2} \mathrm{Zu}$ den kausalen Verhältnissen würden eigentlich dann auch noch die konzessiven und konsekutiven gehören, so z. B. in der Dudengrammatik von 1959 und in der Arbeit zu den deutschen Konnektoren von Blühdorn et al. 2004 (vgl. insbesondere den Beitrag von Waßner 2004). Anders ist hingegen die Perspektive der Grammatik von Zifonun et al. (1997), die als Oberkategorie die konditional fundierten Verhältnisse annimmt, zu denen kausale, konsekutive, konzessive und finale Verhältnisse gehören (vgl. Zifonun et al. 1997: 2290). 
Die Arbeit gliedert sich in drei Abschnitte: Abschnitt 2 illustriert die bei der Zusammenstellung und Analyse des Inventars angewendete Methode; Abschnitt 3 beschreibt die strukturellen Merkmale der im Inventar enthaltenen kausalen Verknüpfungsmittel; Abschnitt 4 fasst die Ähnlichkeiten und Unterschiede zusammen, die sich zwischen den kausalen Verknüpfungsmitteln des Deutschen und Italienischen zeigen.

\section{$2 \quad$ Methode}

Unter Kausalität wird hier - nach Blühdorn (2006: 257) - die Antwort auf "die Frage, warum und wozu Ereignisse und Zustände eintreten" verstanden, wobei Kausalität die Verknüpfung von Ursachen, Motiven und Gründen mit ihren entsprechenden Folgen, Auswirkungen und Effekten bezeichnet. Dieser Definition zufolge betrachte ich als kausal nicht nur jene Sprachstrukturen, die in den traditionellen Grammatiken als kausal angegeben werden, sondern die kausalen und die semantisch verwandten Relationen (vgl. dazu Blühdorn 2010: 219), darunter auch die finalen und konditionalen Strukturen; denn auch diese drücken das Verhältnis zwischen zwei Ereignissen aus, die in kausaler Beziehung zueinander stehen.

In der traditionellen deutschen Grammatikschreibung sowie in der italienischen, die auf das lateinische Beschreibungsmodell zurückreichen, zählen kausale, konditionale und finale Ausdrucksmittel auf der gleichen Ebene wie die übrigen semantisch-funktionalen Verhältnismöglichkeiten (u. a. temporale, lokale, konzessive, konsekutive Verhältnisse) zu drei distinkten grammatischen Bereichen (vgl. z. B. Engel 2009; Duden 2009). Dabei werden kausale, finale und konditionale Ausdrucksmittel unter Berücksichtigung ihres formalen Aspekts nach Wortarten eingeordnet und infolgedessen in verschiedenen Kapiteln beschrieben.

Die vorliegende Beschreibung geht hingegen nicht von der formalen Ebene aus, sondern von der semantisch-funktionalen: Kausalität schließt die kausalen, finalen und konditionalen Verhältnisse der traditionellen Grammatik ein. Semantisch betrachtet, handelt es sich nämlich bei allen drei Verhältnistypen um Relationen zwischen zwei Ereignissen oder Zuständen, wobei ein Ereignis (bzw. Zustand) die Ursache und das (bzw. der) andere die Wirkung ist. Genauer: Finalität ist die Verknüpfung von Handlungen mit Absichten und Zielen, wobei die Handlung, die ausgeführt wird, mit dem Ziel ausgeführt wird, eine bestimmte Wirkung zu erreichen; das Ziel bzw. die Absicht gilt also als Grund für die durchgeführte Handlung. Konditionalität ist die Verknüpfung zweier Ereignisse bzw. Zustände, von denen ein Ereignis bzw. Zustand die Bedingung ist, und das andere Ereignis (bzw. der andere Zustand) die Folge; die Bedingung gilt also als Ursache des Ereignisses, das die Folge ausdrückt.

Von diesem theoretischen Ansatz ausgehend, wird hier zwischen Kausalität im weiteren Sinne und Kausalität im engeren Sinne unterschieden: Kausalität im weiteren Sinne bezeichnet die semantische Oberkategorie der kausalen und semantisch verwandten Relationen, die die grammatischen Kategorien der Kausalität i. e. S., Finalität und Konditionalität beinhaltet. Die Beschreibung der Oberkategorie der Kausalität im weiteren Sinne soll dazu dienen, den versprochenen Überblick über die Ausdrucksmöglichkeiten des Deutschen und des Italienischen zu bieten. Innerhalb der Oberkategorie der Kausalität im weiteren Sinne werden dann die folgenden drei Kategorien unterschieden: die Kausalität i. e. S., die Finalität und die Konditionalität.

Wie sich auch finale und konditionale Verhältnisse als kausal interpretieren lassen, wird noch einmal durch die in der folgenden Tabelle (Tab. 1) angegebenen Umformulierungen exemplifiziert: ${ }^{3}$

\footnotetext{
${ }^{3}$ Fast alle Beispiele im Aufsatz stammen aus den hier betrachteten Grammatiken des Deutschen und des Italienischen. In den Fällen, in denen die Grammatiken kein Beispiel für die beschriebene Struktur lieferten, wurden Beispielsätze durch google.it und google.de im Internet ausgesucht.
} 


\begin{tabular}{|c|c|c|c|}
\hline & Ereignis 1 & Ereignis 2 & Umformulierung im kausalen Sinne \\
\hline \multirow{2}{*}{$\begin{array}{l}\text { Kausalität } \\
\text { i. e. S. }\end{array}$} & Wir bleiben zu Hause & , weil es regnet. & Wir bleiben zu Hause, weil es regnet. \\
\hline & Rimaniamo a casa & perché piove. & $\begin{array}{l}\text { Rimaniamo a casa perché piove (Ursa- } \\
\text { che). }\end{array}$ \\
\hline \multirow[t]{2}{*}{ Finalität } & Wir lernen Deutsch & $\begin{array}{l}\text {, um eine Arbeitsstel- } \\
\text { le im Tourismusbe- } \\
\text { reich zu finden. }\end{array}$ & $\begin{array}{l}\text { Wir lernen Deutsch, weil wir eine Ar- } \\
\text { beitstelle im Tourismusbereich finden } \\
\text { möchten. }\end{array}$ \\
\hline & Studiamo tedesco & $\begin{array}{l}\text { per trovare un posto } \\
\text { di lavoro nel settore } \\
\text { turistico. }\end{array}$ & $\begin{array}{l}\text { Studiamo tedesco perché desideriamo } \\
\text { trovare un posto di lavoro nel settore } \\
\text { turistico. }\end{array}$ \\
\hline \multirow[t]{2}{*}{ Konditionalität } & Wenn es regnet & $\begin{array}{l}\text {, dann wird die Stra- } \\
\text { ße nass. }\end{array}$ & Die Straße wird nass, weil es regnet. \\
\hline & Se piove & la strada si bagna. & La strada si bagna perché piove. \\
\hline
\end{tabular}

Tab. 1: Kausale Interpretation von kausalen i. e. S., finalen und konditionalen Verhältnissen

Aufgrund dieser Auffassung der Kausalität werden also bei der Zusammenstellung des Inventars insbesondere die Kapitel der deutschen und italienischen Grammatiken betrachtet, die die kausalen i.e.S., die finalen und die konditionalen Ausdrucksmittel beschreiben. Innerhalb des jeweiligen semantisch-funktionalen Bereiches werden dann die Ausdrucksmittel nach Wortarten klassifiziert und untereinander kontrastiv verglichen, mit dem Ziel, funktionale und formale Gemeinsamkeiten und Unterschiede zwischen den beiden Sprachsystemen herauszufinden.

Gegenstand der Analyse sind Präpositionen, Konjunktionen, Subjunktionen, Adverbien und Verb- bzw. Satzformen (Verb-Erstsätze, Partizipialsätze, gerundio). In der Analyse sind auch komplexe Verknüpfungsmittel berücksichtigt, die unter dem formalen Aspekt aus mehreren Wörtern bzw. Strukturen bestehen, auf der syntaktisch-funktionalen Ebene jedoch als Konnektoren gelten. Diese sind:

- Präpositionalphrasen, wie aus diesem Grund/per questo motivo, die adverbiale Funktion haben;

- Präpositionalphrasen, Nominalphrasen und Partizipien in Verbindung mit Subjunktionen, wie bspw. unter der Bedingung, dass/a condizione che, Grund dafür, dass/motivo per cui und angenommen, dass/dato che, die subordinierende Funktion haben;

- Präpositionalphrasen in Verbindung mit Präpositionen wie im Falle von/in caso di, die präpositionale Funktion haben.

Hier werden drei Analyseebenen unterschieden: formal, syntaktisch-funktional und semantisch-funktional. aus diesem Grund ist also folgendermaßen analysierbar: auf der formalen Ebene handelt es sich um eine Präpositionalphrase, auf der syntaktisch-funktionalen Ebene um ein Adverbiale und auf der semantisch-funktionalen Ebene um ein kausales i. e. S. Verknüpfungsmittel.

Von meiner Analyse bleiben die Formen der impliziten Kausalität ausgeschlossen. Kausalität kann nämlich sprachlich nicht nur explizit durch kausale Sprachzeichen, sondern auch implizit gekennzeichnet werden - etwa durch die zeitlich-lineare Abfolge der beschriebenen Ereignisse. Aufgrund der gleichen Konzeption von Kausalität als zeitlich-lineare Abfolge zweier miteinander verbundener Ereignisse, wird in diesem Fall das kausale Verhältnis vom Leser auf der semantisch-pragmatischen Ebene erschlossen, d.h. aufgrund seines Weltwissens und mittels seiner Interpretationsfähigkeit. Die Interpretation ist eben dank der chronologischen Linearisierung der beschriebenen Ereignisse möglich: Das - chronologisch gesehen - erste Ereignis ist die Ursache und das andere die Wirkung (Ursache $\rightarrow$ Wirkung), z. B.: 
Es regnet. Wir bleiben zu Hause. $\rightarrow$ Wir bleiben zu Hause, weil es regnet.

Piove. Rimaniamo a casa. $\rightarrow$ Rimaniamo a casa perché piove.

Wir sind zu Hause geblieben. Es regnete. $\rightarrow$ Wir sind zu Hause geblieben, weil es regnete.

Siamo rimasti a casa. Pioveva. $\rightarrow$ Siamo rimasti a casa perché pioveva.

Von der Analyse werden auch die semantisch-lexikalischen Ausdrucksmöglichkeiten ausgeschlossen: In beiden Sprachen kann Kausalität auch durch Verben (dt. verursachen, begründen; it. causare, motivare), Substantive (dt. Grund, Ursache; it. causa, motivazione) und Adjektive (dt. verursacht, begründet; it. causato, motivato) ausgedrückt werden. Diese Ausdrucksmittel werden nur dann genauer betrachtet, wenn sie in komplexen Verbindungsmitteln (z. B. Präpositionalphrasen auch in Verbindung mit Subjunktionen) enthalten sind, z. B.: aus diesem Grund, unter der Bedingung, dass; per questo motivo, a condizione che.

Die Beschreibung erfolgt in drei Schritten:

(1) In einem ersten Schritt werden die gesamten kausalen Strukturen, die in den Grammatiken des Deutschen und des Italienischen enthalten sind, im Allgemeinen beschrieben, wobei ich mich auf die Bestimmung der kausalen i. w. S. Verknüpfungsmöglichkeiten in beiden Sprachen konzentriere, ohne dabei zwischen kausaler i.e.S., finaler und konditionaler Funktion zu unterscheiden, d.h.: abgesehen davon, ob es sich um kausale i. e. S., finale oder konditionale Strukturen handelt. Ziel dabei ist es, aufgrund der in den Grammatikbüchern enthaltenen Strukturen eine Beschreibung der allgemeinen Tendenzen der beiden Sprachsysteme auszuarbeiten, wobei versucht wird, herauszufinden, welche Strukturen für das Deutsche und für das Italienische als typisch gelten können und welche Wortarten die beiden Sprachen gemeinsam haben, um Kausalität auszudrücken.

(2) In einem zweiten Schritt wird die spezifische semantische Funktion der einzelnen Verknüpfungsmittel (kausal i. e. S., final und konditional) berücksichtigt. Hier ist das Ziel jenem des ersten Schrittes ähnlich; darüber hinaus wird aber auf die spezifischen formalen Besonderheiten eingegangen, die in den beiden Sprachen den Ausdruck von kausalen Verhältnissen bei den jeweiligen semantischen Bereichen kennzeichnen. Dabei werde ich zeigen, dass die Gemeinsamkeiten und Unterschiede, die sich aus der Analyse der Grammatiken ergeben, oft keinen Gemeinsamkeiten und Unterschieden zwischen den Sprachen selbst entsprechen. Viele Strukturen, die in den Grammatiken nicht genannt werden, existieren jedoch in den Sprachsystemen des Deutschen und des Italienischen; ähnliches gilt für jene Strukturen, die in den Grammatiken zu verschiedenen Wortarten zugeordnet werden, aber gleiche Funktion im Sprachgebrauch zeigen.

(3) Im letzten Schritt werden Gemeinsamkeiten und Unterschiede zwischen den beiden Sprachsystemen zusammengefasst.

\section{Die Kausalität im weiteren Sinne. Ein Überblick}

Aus einer vergleichenden Übersicht über die kausalen Ausdrucksmittel, die in den Grammatiken des Deutschen und des Italienischen beschrieben werden, erkennt man, dass sich die beiden Sprachen im Allgemeinen der gleichen Wortarten bzw. Strukturen bedienen, um Kausalität auszudrücken:

- Präpositionen:

Er zitterte vor Kälte.

Sono quasi morta dal freddo.

- Konjunktionen: ${ }^{4}$

\footnotetext{
${ }^{4}$ In Wirklichkeit sind Wörter wie infatti, allora, dunque usw. keine Konjunktion. Vgl. dazu 2.1.1.
} 
Ich musste übersetzen, denn Herbert verstand kein Spanisch.

Non posso uscire, infatti non sto bene.

- Subjunktionen in Verbindung mit finiter bzw. infiniter Verbform:

Da seine Lampe noch brennt, arbeitet er wohl noch.

Poiché me lo chiedi, ti dirò tutto.

Wir arbeiten hart, um angenehm zu leben.

Mi alzo per cucire.

- Partizipialstrukturen:

Vom plötzlichen Einbruch der Nacht überrascht, war er ohne Orientierung.

Preoccupato per il suo silenzio, non volle uscire.

- Präpositionalphrasen mit - syntaktisch gesehen - präpositionaler Funktion:

Wie komme ich im Falle eines Streiks von Frankfurt Hbf zum Flughafen?

In caso di sciopero saranno garantiti solo treni a lunga percorrenza.

- Partizipialstrukturen, Präpositionalphrasen und Nominalphrasen in Verbindung mit subordinierenden Sprachzeichen, die subordinierende Funktion haben:

Ich kann das Gerät reparieren, vorausgesetzt, dass ich die nötigen Ersatzteile bekomme.

Dato che questa sarà, molto probabilmente, l'unica parte di tutta la tesi che verrà letta, mi sembra doveroso dedicarle molta attenzione ed il giusto spazio.

Im Fall, dass die elektrischen Leitungen nicht erneuert werden, miete ich diese Wohnung nicht. Nel caso in cui venisse approvata la legge Bavaglio non sarà più possibile leggere questo articolo.

Das ist der Grund, weshalb die meisten Menschen Angst vor ihr haben.

Ed è il motivo per cui molte ditte importanti non sono passate alla vendita diretta al pubblico.

Es gibt jedoch Strukturen, die entweder nur in den deutschen oder in den italienischen Grammatiken vorkommen. Im Deutschen handelt es sich um die uneingeleiteten Nebensätze, die Adverbien ${ }^{5}$ und Subjunktionen in Verbindung mit Korrelatadverbien, ${ }^{6}$ z. B.:

Betrachtet man die aktuellen Forschungstätigkeiten, sind nicht wenige davon durch Vorschläge und Ideen direkt von Studenten entstanden.

Es regnet. Ich bleibe also zu Hause.

In der Antike wurde deshalb gefastet, weil man sich umso weniger Böses einverleibt, je weniger man isst.

Wenn Sie Geld verdienen, dann müssen Sie natürlich auch Einkommensteuer zahlen.

Eine Struktur, die nur in den Grammatiken des Italienischen genannt wird, ist hingegen die Verbform des gerundio: ${ }^{7}$

Essendo arrivati in ritardo, hanno perso il treno.

Aus diesem ersten Überblick über die kausalen Strukturen des Deutschen und des Italienischen ergibt sich, dass es zwischen den beiden Sprachen viele Gemeinsamkeiten, aber auch gewisse Unterschiede gibt. Viele Unterschiede, die in den Grammatiken vorhanden sind, sind jedoch in Wirklichkeit in der Sprache nicht vorhanden. Wie sich die kausalen Strukturen bzw.

\footnotetext{
${ }^{5}$ In Wirklichkeit gibt es auch im Italienischen uneingeleitete Nebensätze und Adverbien. Vgl. dazu 2.1.1; 2.2.3.

${ }^{6} \mathrm{Zu}$ den Korrelatadverbien vgl. Pasch et al. 2003 und Blühdorn 2009. Ähnliche Strukturen existieren auch im Italienischen (vgl. dazu 2.1.1; 2.1.3.).

${ }^{7}$ Obwohl das gerundio eine typisch italienische Struktur ist, finden sich ähnliche Konstruktionen auch im Deutschen. Vgl. dazu 2.1.1.
} 
Wortarten des Deutschen und des Italienischen entsprechen, ist Gegenstand des nachfolgenden Abschnitts zu den einzelnen semantischen Bereichen der Kausalität i. w. S.

\subsection{Zu den einzelnen semantischen Bereichen der Kausalität i. w.S.}

\subsubsection{Kausalität im engeren Sinne}

Aus der Analyse der in den Grammatiken genannten kausalen Strukturen, ergibt sich, dass sowohl das Deutsche als auch das Italienische über eine relativ hohe Anzahl von kausalen i. e. S. Strukturen verfügen. Zwischen den kausalen Ausdrucksmöglichkeiten der beiden Sprachen zeigen sich sowohl Entsprechungen als auch Unterschiede. Das Deutsche sowie das Italienische verwenden Präpositionen, Subjunktionen und Konjunktionen, um Kausalität i. e. S. auszudrücken. Sowohl das Deutsche als auch das Italienische besitzen eine hohe Anzahl an Subjunktionen: Im Deutschen sind dies da, nachdem, nun (da), um so mehr/weniger als, weil und zumal (da), im Italienischen gibt es dacché, giacché, perché, poiché und siccome. Das Deutsche hat auch eine hohe Anzahl von Präpositionen/Postpositionen (an, aufgrund, aus, dank, durch, halber, infolge, mangels, mittels/vermittels, über, unter, von, vor, wegen), während in den italienischen Grammatiken nur drei Präpositionen ( $d a, d i$, per) genannt werden. ${ }^{8}$ Das Italienische verfügt wiederum über eine relativ hohe Anzahl an Konjunktionen (z. B.: pertanto, quindi, allora), während es im Deutschen nur eine Konjunktion (denn) gibt (vgl. Tab. 2):

\begin{tabular}{|l|l|}
\hline Deutsch & Italienisch \\
\hline \multicolumn{1}{|c|}{ Präpositionen/Postpositionen } \\
\hline $\begin{array}{l}\text { an, aufgrund, aus, dank, durch, halber, infolge, } \\
\text { mangels, mittels/vermittels, über, unter, von, vor, } \\
\text { wegen }\end{array}$ & da, di, per \\
\hline \multicolumn{2}{|c|}{ Subjunktionen } \\
\hline $\begin{array}{l}\text { da, weil, zumal }(d a) \text {, nachdem, nun (da), um so } \\
\text { mehr/weniger als, weil, zumal (da) }\end{array}$ & dacché, giacché, perché, poiché, siccome \\
\hline \multicolumn{2}{|c|}{ Konjunktionen } \\
\hline denn & $\begin{array}{l}\text { allora, come, così, dunque, ebbene, infatti, pertan- } \\
\text { to, quindi }\end{array}$ \\
\hline
\end{tabular}

Tab. 2: Kausale (i. e. S.) Wortarten, die sowohl im Deutschen als auch im Italienischen auftreten

Infatti und andere Wörter wie bspw. pertanto, quindi, dunque, allora werden in den italienischen Grammatiken der Wortart Konjunktion zugeordnet; im Sprachgebrauch fungieren sie allerdings eher als Adverbien. Renzi/Salvi/Cardinaletti (2001: 245) erkennen, dass sie einen Sonderfall darstellen, und nennen diese "Pseudo-Konjunktionen". 9 Im Italienischen gibt es also vermutlich keine Konjunktion, die der deutschen Konjunktion denn entspricht.

Andere Strukturen werden hingegen entweder nur in den deutschen oder in den italienischen Grammatiken genannt; es ist trotzdem möglich, Entsprechungen zwischen den Ausdrucksmöglichkeiten des Deutschen und jenen des Italienischen zu finden. Für das Deutsche sind dies:

- Adverbien: also, folglich, insofern, mithin, nämlich, und Pronominaladverbien: daher, darum, demnach, deshalb, deswegen, infolgedessen, somit;

\footnotetext{
${ }^{8}$ Im Italienischen gibt es keine Postpositionen; es sind dies typisch deutsche Strukturen.

${ }^{9}$ Von hier an werden diese italienischen Konjunktionen "Pseudo-Konjunktionen" genannt.
} 
- Subjunktionen in Verbindung mit fakultativen Adverbien, z. B.: weil/deswegen, deshalb/weil;

- Präpositionalphrasen mit adverbialer Funktion, z. B.: aus diesem Grund;

- Fragepronomina mit subordinierender Funktion, die als Relativpronomina verwendet werden, z. B.: warum, weshalb, weswegen, wieso, wo;

- Nominalphrasen in Verbindung mit Pronominaladverbien und Subjunktion, z. B.: die Begründung dafür, dass oder in Verbindung mit Relativpronomina, z. B.: der Grund, warum und die Ursache, weshalb, die als subordinierende Verknüpfungsmittel dienen.

Im Italienischen gibt es einzelne Formen, die als Äquivalente dieser Konstruktionen angesehen werden können; diese Formen werden von den italienischen Grammatiken jedoch nicht in allen Fällen als Mittel zum Ausdruck von Kausalität klassifiziert. Auch im Italienischen besteht z. B. die Möglichkeit, kausale Adverbien von einem Nomen abzuleiten; insbesondere finden die Adverbien also und folglich eine Entsprechung im vom Adjektiv conseguente ${ }^{10}$ (dt. folglich) abgeleiteten Adverb conseguentemente:

Erwartet wird ein Dreikampf um das Tor - also steht Kessler im direkten Konkurrenzkampf neben Hain und Bene um den Platz zwischen den Pfosten.

Es ist geschehen, und folglich kann es wieder geschehen.

Come si sa, il costo del petrolio e conseguentemente dei carburanti nel periodo estivo decollano tangenzialmente [...].

Im Allgemeinen finden die deutschen Adverbien im Italienischen in Strukturen eine Entsprechung, die zu anderen Wortarten gehören:

1. die Adverbien wie also und nämlich entsprechen Pseudo-Konjunktionen wie dunquelquindi und infatti/pertanto, z. B.:

Monika sagt, sie sei krank. Sie kommt heute also nicht.

Ho la febbre; quindi non vado a scuola.

Die angegebenen Beispiele bestätigen, dass es tatsächlich eine funktionale Entsprechung zwischen den deutschen Adverbien und den italienischen Pseudo-Konjunktionen gibt: Obwohl infatti, pertanto, allora üblicherweise in den italienischen Grammatiken nicht als Adverbien, sondern als Konjunktionen klassifiziert werden, ist hier ihre semantische Funktion mit jener der deutschen kausalen Adverbien identisch.

2. die Pronominaladverbien des Deutschen, die in der italienischen Grammatikschreibung nicht vorkommen, können im Italienischen Präpositionalphrasen wie a causa di ciò, per questo motivo u. ä. entsprechen, z. B.:

Es regnete, darum blieb er zu Hause.

Ho la febbre. Per questo motivo non vado a scuola.

Obwohl Pronominaladverbien in den italienischen Grammatiken nicht genannt werden, existieren sie in der italienischen Sprache. Als Beispiel dafür gilt perciò: Traditionell wird perciò zur Wortart Konjunktion zugeordnet, morphologisch und syntaktisch ist es aber ein Pronominaladverb:

Ho la febbre, perciò non vado a scuola.

Nominalphrasen in Verbindung mit Pronominaladverbien und Subjunktionen, Relativpronomina und Nominalphrasen in Verbindung mit Relativpronomina finden im Italienischen eine

\footnotetext{
10 Das Adjektiv conseguente ist vom Substantiv conseguenza (dt. Folge) abgeleitet.
} 
Entsprechung in ähnlichen Ausdrucksmitteln, die aber eine andere innere formale Struktur als die deutschen aufweisen:

In seinem Artikel ("Europa als Lebensform", S. 53) liefert Hans Ulrich Gumbrecht so quasi die Begrïndung dafür, dass es in Europa (will heissen: in der EU) so weit kommen konnte. $\grave{E}$ stata inventata la macchina che scopre la causa per cui non riusciamo a dormire.

Manches wäre noch zu nennen, weswegen man sich kritische Gedanken machen müsste. Verranno tempi migliori. Questo è il motivo per il quale continuo la mia ricerca.

Der Grund, warum manche Mitarbeiter auf der Leiter des Erfolges nicht so recht vorankommen, ist darin zu suchen, dass sie glauben, sie stünden auf einer Rolltreppe.

Questo è il motivo per cui molte ditte hanno chiuso i battenti.

C'è un motivo perché piova sempre nel weekend?

Die angegebenen Beispiele zeigen, dass die Strukturen des Deutschen unter funktionalem Aspekt jenen des Italienischen äquivalent sind. Unter formalem Aspekt sind sie hingegen ähnlich, aber nicht miteinander identisch: Komplexe Strukturen wie die Begründung dafür, dass oder der Grund, warum des Deutschen und komplexe Strukturen wie il motivo per cui, il motivo perché, la causa per cui des Italienischen bestehen aus einer Nominalphrase und einem subordinierenden Sprachzeichen (Subjunktion bzw. Relativpronomen), dessen syntaktische Funktion es ist, einen Nebensatz einzuleiten. Als Beispiel dafür gilt der Vergleich zwischen der Verbindung Begründung dafür, dass des Deutschen und der Verbindung il motivo per cui des Italienischen. In il motivo per cui ist das Relativpronomen cui durch die Präposition per eingeleitet, welche dem im deutschen Pronominaladverb dafür enthaltenen Wort für entspricht. Als Subordinationszeichen dient im Deutschen die neutrale Subjunktion dass, während die Beziehung zwischen Nominalphrase und Nebensatz im Italienischen durch das Relativpronomen cui markiert wird, wobei cui allerdings eine unmarkierte Pronominalform ist. Die Form cui gilt im Italienischen als Korrelat des Substantivs motivo - sowie da des Wortes dafür des Deutschen Korrelat des Substantivs Begründung ist - und leitet als Subjunktor den Nebensatz ein, weswegen sich eine Doppelentsprechung ergibt (da/dass $\rightarrow$ cui). Schematisch lassen sich die formalen und syntaktisch- bzw. semantisch-funktionalen Entsprechungen zwischen der deutschen und italienischen Struktur folgendermaßen darstellen:

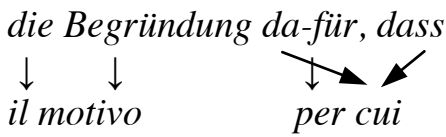

Andere Strukturen werden nur in den italienischen Grammatiken genannt:

- Partizipialstrukturen (auch in Verbindung mit einer Subjunktion): dato che, visto che;

- Präpositionalphrasen in Verbindung mit einer Subjunktion: per il fatto che, per il motivo che;

- Präpositionalphrasen in Verbindung mit einer Präposition: a causa di, per ragioni di;

- die Subjunktion per in Verbindung mit einem Infinitiv in der Vergangenheitsform.

Im Deutschen existieren jedoch entsprechende Ausdrucksmöglichkeiten:

- Präpositionen:

Dato il grande numero di richieste, le domande devono essere presentate con 2-3 mesi di anticipo.

Wegen der hohen Geschwindigkeit der Moleküle und wegen der großen Zahl von Stößen können die Moleküle in einem Gas nicht aneinander haften bleiben.

- Satzstrukturen mit einem durch Subjunktion eingeleiteten Nebensatz und finitem Verb:

Visto che le tasse non si possono tagliare, il governo le aumenta. 
Da die Wetter- und vor allem Windfestigkeit auch erheblich vom verwendeten Gestänge abhängt, sollte man hier auf jeden Fall von Fiberglasgestängen Abstand nehmen [...].

$\grave{E}$ stato sgridato per essere arrivato in ritardo.

Nur zwei Kinder wurden gescholten, weil sie sich verspätet hatten.

- Präpositionalphrasen:

Assente per ragioni di servizio.

Aus Sicherheitsgründen gesperrt.

Die Verbindung Adv/Subj bzw. Subj/Adv wird nur in den deutschen Grammatiken genannt. Im Italienischen existieren jedoch entsprechende Strukturen: als Korrelat der Subjunktion kommen die sogenannten Pseudo-Konjunktionen vor:

Vielleicht war Marie deshalb nicht mit mir nach Rom gefahren, weil sie sich dort ihres sündigen Zusammenlebens mit mir besonders hätte schämen müssen.

Poiché il tema era difficile, così non l'ho potuto svolgere.

Siccome mi sentivo male, allora sono stato dal medico.

Giacché non è possibile, allora è meglio non tentare.

Typisch italienisch scheint die Verbform des gerundio zu sein, die als grammatische Struktur in den deutschen Grammatiken nicht genannt wird und im Deutschen in anderen Strukturen eine Entsprechung findet, wie bspw. in durch Subjunktion eingeleiteten Nebensätzen mit finiter Verbform, in Präpositionen oder Pronominaladverbien:

Non avendo denaro, non sono potuto andare al concerto.

Da ich keinen Hunger habe, bestelle ich nur einen Kaffee.

Wegen Geldmangels musste nun auch das letzte Oberhausener Arbeitslosenzentrum schließen.

Er hatte kein Geld, und deshalb ging er nicht in Urlaub.

Wenn man aber betrachtet, dass die grammatische und semantische Funktion des gerundio genau wie jene eines Partizips ist, dann können die romanischen Gerundialkonstruktionen im Deutschen jederzeit durch Partizipialkonstruktionen wiedergegeben werden, die typisch für die Schriftsprache sind: Zu spät angekommen, haben sie den Zug verpasst.

\subsubsection{Finalität}

Sowohl das Deutsche als auch das Italienische verfügen über eine geringe Menge von Strukturen, die zum Ausdruck der finalen Verhältnisse dienen, wobei das Italienische aber mehr Strukturen als das Deutsche zählt, wie in der folgenden Tabelle veranschaulicht wird (vgl. Tab. 3):

\begin{tabular}{|l|l|l|}
\hline Deutsch & Italienisch \\
\hline \multicolumn{2}{|c|}{ Subjunktionen mit finiter Verbform } \\
\hline $\begin{array}{l}\text { damit, auf dass (gehoben, veraltend), dass (nur } \\
\text { alltagssprachlich) }\end{array}$ & affinché, che, perché \\
\hline \multicolumn{2}{|c|}{ Subjunktionen mit infiniter Verbform } \\
\hline$u m(z u)$ & per \\
\hline$z u, z w e c k s$ & Präpositionen \\
\hline \multicolumn{2}{|c|}{} & $a, d a$, in, per \\
\hline
\end{tabular}

Tab. 3: Finale Wortarten, die sowohl im Deutschen als auch im Italienischen auftreten

Zum Ausdruck der Finalität dienen in beiden Sprachen Subjunktionen mit finiter Verbform, Subjunktionen mit infiniter Verbform und Präpositionen: 


\section{Manch einer klagt, damit man ihn bedauert.}

Studiate affinché siate promossi.

Es soll ein Studienaufenthalt zum Deutschlernen werden.

Lottare per il successo.

Ich kaufe mir eine Brille, um besser zu sehen.

Mi alzo per cucire.

Bei den einzelnen Wortarten sind unter formalem Aspekt sowohl Ähnlichkeiten als auch Unterschiede zwischen den beiden Sprachen zu beobachten. Die Subjunktionen des Deutschen sind auf dass (gehoben, veraltet), damit, dass (nur alltagssprachlich) und um zu; jene des Italienischen sind affinché, che, perché und per. Sowohl im Deutschen als auch im Italienischen bestehen die meisten Subjunktionen aus einem subordinierenden Sprachzeichen dass bzw. che (auf dass und dass für das Deutsche; affinché, perché und che für das Italienische), das sich in all seinen Realisierungen durch das Merkmal Neutralität ähnelt. Bei auf dass, a che (veraltet), accioché (veraltet), affinché und perché steht dem subordinierenden Sprachzeichen eine Präposition vor: auf im Deutschen, $a$ und per im Italienischen, die aus dem räumlichen Bereich stammen. Anders gelagert ist der Fall der Subjunktion damit, einer Zusammensetzung des Adverbs $d a$ mit der Präposition mit, bei instrumentaler Herkunft.

Eine andere Ähnlichkeit ergibt sich bei den Subjunktionen in Verbindung mit Infinitivformen: Diese entsprechen einander in den beiden Sprachen nicht nur deswegen, weil sie eine infinite Verbform einleiten, sondern auch, weil sie aus der Wortart Präposition stammen: $u m$ und $z u$ im Deutschen, per im Italienischen.

Für die Präpositionen gelten hingegen folgende Beobachtungen: Das Deutsche hat nur zwei Präpositionen: $z u$ und zwecks, das Italienische vier: $a$, in, per, $d a$. Die deutsche Präposition $z u$ und alle italienischen Präpositionen kommen aus dem räumlichen Bereich. Im Deutschen gibt es darüber hinaus die Präposition zwecks, welche eine Ableitung aus dem Substantiv Zweck ist und keine direkte Entsprechung im Italienischen findet. Im Italienischen gibt es jedoch ähnliche Präpositionalverknüpfungen, wobei die Substantive scopo und fine (dt. Zweck/Ziel) in Präpositionalphrasen wie a scopo di, al fine di auftreten.

Wie bei der Kausalität i. e.S. gibt es auch bei der Finalität Strukturen, die entweder nur im Deutschen oder im Italienischen vorkommen. Im Deutschen gilt dies für das Pronominaladverb dazu:

Ein Picknick im Freien. Was brauche ich dazu?

Das Italienische besitzt kein finales Adverb: dazu kann hier in einer Präpositionalphrase, d.h. in einer analytischen Struktur wie a tale scopo, a tal fine, eine Entsprechung finden, z. B.:

Il sottoscritto chiede di essere esonerato dal pagamento delle tasse. A tal fine dichiara quanto segue: [...].

Typisch allein für das Italienische sind hingegen die Präpositionalphrasen in Verbindung mit einer Subjunktion:

Una buona poltrona deve permettere di regolare anche l'altezza dei poggia braccia, di modo che le braccia possano riposare nella posizione corretta.

Obwohl es sich formal um eine Präpositionalphrase in Verbindung mit einer Subjunktion handelt, fungiert di modo che unter dem funktionalen Aspekt als eine Subjunktion (wie beispielsweise affinché). 


\subsubsection{Konditionalität}

Sowohl im Deutschen als auch im Italienischen sind die Strukturen, die zum Ausdruck der Konditionalität dienen, sehr vielfältig. In beiden Sprachen sind dies Subjunktionen, Präpositionen, Partizipialstrukturen (auch in Verbindung mit Subjunktionen) und Präpositionalphrasen in Verbindung mit Subjunktionen (vgl. Tab. 4):

\begin{tabular}{|c|c|}
\hline Deutsch & Italienisch \\
\hline \multicolumn{2}{|c|}{ Subjunktionen } \\
\hline falls, sofern, soweit, wenn, wenn... schon & qualora, se, affinché, che, perché \\
\hline \multicolumn{2}{|c|}{ Präpositionen } \\
\hline bei & con, $d a$ \\
\hline \multicolumn{2}{|c|}{ Partizipialstrukturen (auch in Verbindung mit Subjunktionen) } \\
\hline $\begin{array}{l}\text { gesetzt den Fall, vorausgesetzt, vorausgesetzt, } \\
\text { dass }\end{array}$ & considerato il caso, considerato che, supposto che \\
\hline \multicolumn{2}{|c|}{ Präpositionalphrasen in Verbindung mit Subjunktionen } \\
\hline $\begin{array}{l}\text { im Fall(e), dass, unter der Bedingung, dass, unter } \\
\text { der Voraussetzung, dass }\end{array}$ & a condizione che, a meno che \\
\hline
\end{tabular}

Tab. 4: Konditionale Wortarten, die sowohl im Deutschen als auch im Italienischen auftreten Beispiele:

Bei schlechtem Wetter (bei Regen, bei Wind, bei Sturm) arbeite ich am liebsten.

Con quel buio non potevamo vedere.

Man braucht eine Steuerkarte, wenn man eine Arbeit annimmt.

Se vuoi, t'aiuto.

Gesetzt den Fall, dass Sie mich sprechen wollen, rufen Sie mich bitte vorher an!

Considerato che non ci sono politici né dirigenti onesti cosa si deve fare per risollevare l'Italia?

Ich helfe dir nur unter der Bedingung, dass du mir auch hilfst.

Io verrò a condizione che tu ci sia.

Verb-Erstsätze und Subjunktionen in Verbindung mit einem Korrelatadverb werden nur in den deutschen Grammatiken genannt:

Betrachtet man aus ca. $15 \mathrm{~cm}$ Entfernung mit dem linken Auge (rechtes Auge geschlossen) das

Kreuz auf der Abbildung, verschwindet der Kreis, da dieser Bereich an der Stelle des "Blinden

Flecks" auf die Netzhaut projiziert wird.

Wenn Sie Geld verdienen, dann müssen Sie natürlich auch Einkommensteuer zahlen.

In den italienischen Grammatiken werden keine Verb-Erstsätze beschrieben, die kausale Interpretation haben können; sie entsprechen Sätzen, die durch eine Subjunktion eingeleitet sind oder durch gerundio-Konstruktionen ausgedrückt wird:

Se arrivi in tempo sulla palla, questa approda facilmente nell'altra metà-campo.

Arrivando in ritardo sulla palla, questa approda facilmente nell'altra metà campo.

In Wirklichkeit gibt es auch im Italienischen uneingeleitete Nebensätze, z. B.:

Dovesse piovere, preferisco non uscire.

Den Subjunktionen in Verbindung mit Adverbien entsprechen im Italienischen wie bei der Kausalität i. e. S. Subjunktionen in Verbindung mit Konjunktionen, z. B.: 


\section{Se $x \grave{e ̀ ~ u n ~ u o m o, ~ a l l o r a ~} x \grave{e ̀ ~ m o r t a l e . ~}$}

Die Unterschiede zwischen den deutschen und den italienische Bildungen, die eine solche Struktur zeigen, wurden schon für die Kausalität i. e. S. besprochen. Hier gelten die gleichen Bemerkungen.

In den italienischen Grammatiken werden keine konditionalen Wortarten genannt, die in den deutschen nicht vorkommen.

\section{$4 \quad$ Fazit}

Aus der Analyse der Grammatiken der deutschen und der italienischen Sprache lassen sich viele Ähnlichkeiten zwischen dem deutschen und dem italienischen Repertoire von kausalen Strukturen beobachten; es gibt allerdings auch viele Unterschiede. Einige Wortarten bzw. Strukturen werden in den Grammatiken der beiden Sprachen genannt, während andere nur in den deutschen oder in den italienischen Grammatiken vorkommen. Aufgrund der grammatischen Beschreibungen wären die gemeinsamen Wortarten bzw. Strukturen folgende: Präpositionen, Konjunktionen, Subjunktionen in Verbindung mit finiter oder infiniter Verbform, Partizipialstrukturen und andere komplexe Verbindungsmittel wie bspw. Präpositionalphrasen oder Nominalphrasen in Verbindung mit einem subordinierenden Sprachzeichen. Andere Strukturen werden hingegen entweder nur in den deutschen Grammatiken oder in den italienischen genannt. Dies sind die Verb-Erstsätze und die Adverbien/Pronominaladverbien für das Deutsche und das gerundio für das Italienische. Betrachtet man aber die Sprachen selbst, dann sind nicht alle diese Unterschiede vorhanden: Das Deutsche hat Partizipialstrukturen, die formal und syntaktisch dem gerundio ähnlich sind; Verb-Erstsätze gibt es auch im Italienischen; das Italienische besitzt außerdem Pseudo-Konjunktionen (z. B. pertanto, perciò, allora, infatti), die auf der syntaktischen Ebene wie Adverbien fungieren. $\mathrm{Zu}$ den echten Unterschieden gehören vielmehr folgende zwei Punkte:

1) Die Wortart Konjunktion tritt nur im Deutschen auf, und zwar im kausalen Bereich. Es handelt sich um die Konjunktion denn. Das Italienische scheint keine direkte Entsprechung zu haben.

2) Alle den beiden Sprachen gemeinsame Wortarten bzw. Strukturen unterscheiden sich von Sprache zu Sprache durch ihre innere Struktur und sind in den beiden Sprachen in den drei kausalen Bereichen mit verschiedener Häufigkeit und Vielfältigkeit verteilt:

a) Der Bereich, in dem die größte Anzahl von Strukturen vorkommt, ist sowohl im Deutschen als auch im Italienischen jener der Kausalität i. e. S., bei dem die Varietät der Verknüpfungsmittel nicht nur numerisch ist, sondern auch ihre innere formale Struktur betrifft. Im Deutschen sind die reichsten Klassen jene der Subjunktionen, Präpositionen und jene der Adverbien, im Italienischen jene der Pseudo-Konjunktionen und der Subjunktionen. Die deutschen Adverbien und die italienischen Pseudo-Konjunktionen sind ferner durch ähnliche Wortbildungsverfahren charakterisiert. Die meisten deutschen Adverbien sind Pronominaladverbien, die aus dem lokalen Adverb da und einer lokalen Präposition bzw. Partikel bestehen: dar+um, da+her, wodurch die Herkunft (Grund, Ursache) angezeigt wird, woher etwas (eine Wirkung) kommt, oder aus dem bestimmten Artikel im Genitiv und einer Präposition des+halb, des+wegen. In anderen Fällen werden Präpositionen mit Nomina zusammengesetzt: $z u+f o l g e$, in folge. Ähnliche Verfahren kommen bei den italienischen Pseudo-Konjunktionen vor: Präposition + Adjektiv (per+tanto), Konjunktion + Adjektiv $(e(b)+b e n e)$, Präposition + Nomen in+fatti. Unterschiede kommen bei der Kausalität in den Fällen vor, in denen das Italienische infinite Verbformen verwendet (z. B.: gerundio, Partizip). Hier hat das Italienische die Tendenz, implizit zu sein, während das Deutsche die kausalen Verhältnisse durch Präpositionen und Konjunktionen expliziert. 
b) Bei der Finalität treten hingegen sowohl im Deutschen als auch im Italienischen weniger Verknüpfungsmittel auf: Dabei gibt es in Bezug auf die innere formale Struktur eine direktere Entsprechung zwischen den Verknüpfungsmitteln des Deutschen und jenen des Italienischen. Die beiden Sprachen unterscheiden sich hier nur deswegen, weil das Deutsche das Adverb dazu und die Präposition zwecks als typische Strukturen hat, während die typische Struktur des Italienischen die Präpositionalphrase in Verbindung mit einer Subjunktion ist (in modo che), die jedoch subordinierende Funktion hat und mit der deutschen Subjunktion damit zusammenfällt.

c) Bei der Konditionalität gibt es auch vor allem Gemeinsamkeiten zwischen dem Deutschen und dem Italienischen: Die Verknüpfungsmittel der Konditionalität entsprechen einander besser als jene der anderen Bereiche. Die Unterschiede betreffen eher die Fachterminologie, d.h. die Art und Weise, wie Wortarten in den beiden Grammatikschreibungstraditionen klassifiziert werden: Als Beispiel dafür gelten die komplexen Sätzen, die im Deutschen eine Korrelation zwischen Subjunktion (im Nebensatz) und Adverb (im Hauptsatz) aufweisen, während im Italienischen die Korrelation zwischen Subjunktion und PseudoKonjunktion erfolgt.

Die durchgeführte Analyse bestätigt, dass die Verwendung der kontrastiven Methode ein hilfreiches Mittel im Grammatikunterricht bei DaF-Lernern sein kann, um einen Überblick über die deutsche (Ziel)grammatik zu erhalten. Eine genauere Beschreibung der morphosyntaktischen Gemeinsamkeiten und Unterschiede zwischen den deutschen und italienischen Strukturen bleibt hingegen noch ein Desiderat für zukünftige Untersuchungen.

\section{Literatur}

Ballestracci, Sabrina (2009): "Leseverstehensstrategien bei der Analyse kausaler Strukturen im theoretischen Kurs Lingua Tedesca 1". In: Foschi Albert, Marina/Hepp, Marianne (eds.): Texte - Lesen. Ansichten aus der polnischen und italienischen DaF-Didaktik. Pisa, Jacques e i suoi quaderni: 160-169.

Battaglia, Salvatore/Pernicone, Vincenzo (1994): Grammatica italiana. Torino: Loescher.

Blühdorn, Hardarik (2006): Kausale Satzverknüpfungen im Deutschen. São Paulo, Pandaemonium Germanicum: 253-282. (= Revista de Estudos Germanísticos 10).

Blühdorn, Hardarik (2010): "A semantic typology of sentence connetives". In Harden, Theo/Hentschel, Elke (eds.): 40 Jahre Partikelforschung. Tübingen, Stauffenburg: 215-231.

Brdar-Szabó, Rita (2001): "Kontrastivität in der Grammatik". In Helbig, Gerhard/Götze, Lutz/Heinrichi, Gert/Krumm, Jürgen (ed.). Deutsch als Fremdsprache. Ein internationales Handbuch. Berlin/New York, de Gruyter: 195-204.

Dardano, Maurizio/Trifone, Pietro (1995): Grammatica italiana: con nozioni di linguistica. 3. Aufl. Bologna: Zanichelli.

Diehl, Erika et al. (2000): Grammatikunterricht: Alles für der Katz? Untersuchungen zum Zweitsprachenerwerb Deutsch. Tübingen: Niemeyer.

Duden (2009): Die Grammatik. 8., überarb. Aufl. Mannheim/Leipzig/Wien/Zürich: Duden.

Eisenberg, Peter (2006): Grundriß der deutschen Grammatik. 2., überarb. und aktual. Aufl. Stuttgart/Weimar: Metzler.

Engel, Ulrich (2009): Deutsche Grammatik. Neubearb. 2., durchges. Aufl. Heidelberg: Gross.

Pasch, Renate et al. (2003): Handbuch der deutschen Konnektoren. Linguistische Grundlagen der Beschreibung und syntaktische Merkmale der deutschen Satzverknüpfer (Konjunktionen, Satzadverbien und Partikeln). Berlin: de Gruyter.

Rall, Marlene (2001): "Grammatikvermittlung". In Helbig, Gerhard/Götze, Lutz/Heinrichi, Gert/Krumm, Jürgen (ed.): Deutsch als Fremdsprache. Ein internationales Handbuch. Berlin/New York, de Gruyter: 880-886. 
Ravetto, Miriam/Blühdorn, Hardarik (2011): "Die Kausalkonjunktionen denn, weil, da im Deutschen und perché, poiché, siccome im Italienischen". In: Ferraresi, Gisella (ed.): Konnektoren im Deutschen und im Sprachvergleich. Beschreibung und grammatische Analyse. Tübingen, Narr: 207-250.

Renzi, Lorenzo/Salvi, Gianpaolo/Cardinaletti, Anna (2001): Grande grammatica italiana di consultazione. Band 1. Bologna: Il Mulino.

Serianni, Luca (1991): Grammatica italiana: italiano comune e lingua letteraria. 2. Aufl. Torino: UTET.

Terrasi-Haufe, Elisabetta (2004): Der Schulerwerb von Deutsch als Fremdsprache. Eine empirische Untersuchung am Beispiel der italienischsprachigen Schweiz. Tübingen: Niemeyer.

Waßner, Ulrich Hermann (2004): "Einleitung". In: Blühdorn, Hardarik/Breindl, Eva/Waßner, Ulrich Hermann (eds.): Brücken schlagen. Grundlagen der Konnektorensemantik. Berlin, de Gruyter: 312-322.

Weinrich, Harald (1993): Textgrammatik der deutschen Sprache. Mannheim/Leipzig/Wien/Zürich: Duden.

Zifonun, Gisela et al. (1997): Grammatik der deutschen Sprache. Berlin: de Gruyter. 